\title{
Charge accumulation at organic semiconductor interfaces due to a permanent dipole moment and its orientational order in bilayer devices
}

Cite as: J. Appl. Phys. 111, 114508 (2012); https://doi.org/10.1063/1.4724349

Submitted: 21 March 2012 . Accepted: 30 April 2012 . Published Online: 05 June 2012

Yutaka Noguchi, Yukimasa Miyazaki, Yuya Tanaka, Naoki Sato, Yasuo Nakayama, Tobias D. Schmidt, Wolfgang Brütting, and Hisao Ishii

ARTICLES YOU MAY BE INTERESTED IN

Influence of the direction of spontaneous orientation polarization on the charge injection properties of organic light-emitting diodes

Applied Physics Letters 102, 203306 (2013); https://doi.org/10.1063/1.4807797

Nonradiative recombination centers and electrical aging of organic light-emitting diodes:

Direct connection between accumulation of trapped charge and luminance loss

Journal of Applied Physics 93, 1108 (2003); https://doi.org/10.1063/1.1531231

Organic electroluminescent diodes

Applied Physics Letters 51, 913 (1987); https://doi.org/10.1063/1.98799

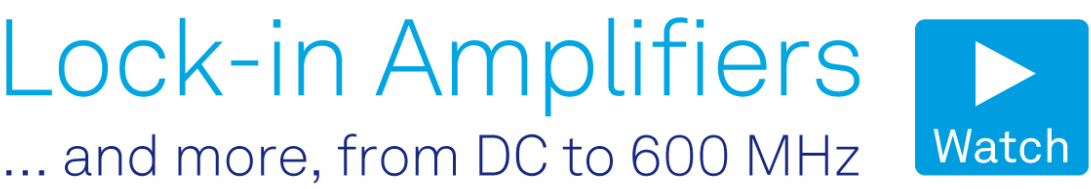




\title{
Charge accumulation at organic semiconductor interfaces due to a permanent dipole moment and its orientational order in bilayer devices
}

\author{
Yutaka Noguchi, ${ }^{1,2,3, a)}$ Yukimasa Miyazaki, ${ }^{2}$ Yuya Tanaka, ${ }^{2}$ Naoki Sato, ${ }^{2}$ Yasuo Nakayama, ${ }^{1}$ \\ Tobias D. Schmidt, ${ }^{4}$ Wolfgang Brütting, ${ }^{4}$ and Hisao Ishii ${ }^{1,2, b)}$ \\ ${ }^{1}$ Center for Frontier Science, Chiba University, 1-33 Yayoi-cho, Inage, Chiba 263-8522, Japan \\ ${ }^{2}$ Graduate School of Advanced Integration Science, Chiba University, 1-33 Yayoi-cho, Inage-ku, \\ Chiba 263-8522, Japan \\ ${ }^{3}$ PRESTO, Japan Science and Technology Agency, 4-1-8 Honcho Kawaguchi, Saitama 332-0012, Japan \\ ${ }^{4}$ Institute of Physics, University of Augsburg, Augsburg 86159, Germany
}

(Received 21 March 2012; accepted 30 April 2012; published online 5 June 2012)

\begin{abstract}
Charge accumulation at the organic heterointerfaces in multilayer organic light-emitting diodes (OLEDs) is an important process for understanding their device operation, efficiency, and degradation properties. Charge accumulation behavior has typically been analyzed in terms of the energy barrier and difference of the charge carrier mobility across heterointerfaces. In this study, we demonstrate that permanent dipole moments and their orientational order also play a significant role in the charge behavior at organic semiconductor interfaces. The charge accumulation properties of bilayer devices composed of polar or nonpolar molecules deposited on a 4,4'-bis[ $N$-(1-naphthyl)- $N$-phenylamino] -biphenyl layer between the anode and cathode were examined by displacement current measurement and impedance spectroscopy. In addition, Kelvin probe measurements for the corresponding bilayer structures excluding the cathode were performed to analyze the relationship between the potential profile and charge accumulation properties of the bilayer devices. We found that several polar molecules including tris-(8-hydroxyquinolate) aluminum, 1,3,5-tris(1-phenyl-1 H-benzimidazol-2-yl) benzene, 2,9-dimethyl-4,7-diphenyl-1,10-phenanthroline (BCP), and 1,3-bis[2-(4-tert-butylphenyl)-1, 3,4-oxadiazo-5-yl]benzene (OXD-7) are spontaneously ordered in evaporated films, and orientation polarization remains in bilayer devices. Orientation polarization leads to interface charge which determines the least amount of accumulated charge in the device under operation. The estimated interface charge density for these molecules ranged from -2.3 (OXD-7) to -0.5 (BCP) $\mathrm{mC} / \mathrm{m}^{2}$. Furthermore, impedance spectroscopy revealed that the presence of a permanent dipole moment can suppress the charge dispersion along the interface probably owing to the microscopic potential fluctuation formed in the vicinity of the interface. These results indicate that the permanent dipole moment and orientation polarization contribute to the efficient charge accumulation at organic heterointerfaces and are important parameters for understanding and controlling the charge carrier dynamics in multilayer OLEDs. ( 2012 American Institute of Physics.
\end{abstract}

[http://dx.doi.org/10.1063/1.4724349]

\section{INTRODUCTION}

Charge accumulation behavior at organic heterointerfaces in multilayer organic light-emitting diodes (OLEDs) has been extensively studied using various experimental methods and theoretical models, because understanding their behavior is essential for improving device performance. ${ }^{1-6}$ Accumulated charges can serve to tune the electric field distribution to achieve better charge balance. Moreover, accumulated charges in the emission layer of OLEDs increase the probability of exciton formation, thus, leading to better efficiency. ${ }^{1,2}$ However, in tris-(8-hydroxyquinolate) aluminum $\left(\mathrm{Alq}_{3}\right.$ )-based OLEDs, the charged molecules accumulated near the emission region can also act as exciton quenchers, ${ }^{6,7}$ and the presence of the $\mathrm{Alq}_{3}$ and 4,4'-bis [ $N$-(1-naphthyl)- $N$-phenylamino]-biphenyl $(\alpha-\mathrm{NPD})$ cation species is considered responsible for device degradation. ${ }^{8,9}$

\footnotetext{
${ }^{\text {a)} E l e c t r o n i c ~ m a i l: ~ y-n o g u c h i @ f a c u l t y . c h i b a-u . j p . ~}$

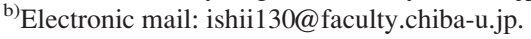

Therefore, detailed understanding of charge accumulation behavior is required to develop highly efficient multilayer OLEDs with appropriately constructed interfaces.

Four elements related to charge accumulation in multilayer OLEDs should be considered: (i) energy barrier, (ii) mobility mismatch, (iii) charge recombination ratio, and (iv) space charge. ${ }^{2,10}$ Here, the space charge includes real and polarization charges. Polarization charges are typically induced by external electric fields and commonly appear as the dielectric constant in the device parameter. In addition, if a device contains polar molecules, contributions from their permanent dipole moments should be considered. In fact, numerous molecules used in organic semiconductor devices possess a permanent dipole moment, and the effects of the dipole moment have been studied in terms of the charge transport properties of amorphous films containing such polar molecules. The presence of dipolar disorder leads to lower carrier mobility in a film because dipole moments induce different local electric fields and thus increase the width of density of states. ${ }^{11,12}$ A similar effect has been 
reported in the transport properties of organic field effect transistors (OFETs), in which the dipolar disorder in the gate dielectric material results in lower charge carrier mobility of the adjacent organic semiconductor film. ${ }^{13-15}$ In addition to these effects of individual dipole moments, contributions from their assembly, such as orientation polarization in a film, are essential for the comprehensive understanding of charge accumulation properties; however, compared to other parameters, they have not been studied sufficiently.

Recently, we proposed that interface charge in an $\mathrm{Alq}_{3}$-based OLED originates from the orientation polarization in the $\mathrm{Alq}_{3}$ film. ${ }^{16}$ Interface charge was first detected by Brütting et al. at the $\alpha-\mathrm{NPD} / \mathrm{Alq}_{3}$ interface in the bilayer OLED. ${ }^{5,17}$ They found that the hole injection voltage $\left(V_{\text {inj }}\right)$ of these devices appears lower than the built-in voltage $\left(V_{\mathrm{bi}}\right)$, and it shifts to the negative side with increasing $\mathrm{Alq}_{3}$ film thickness. This behavior is well explained by assuming a constant amount of negative fixed charge at the $\alpha-\mathrm{NPD} / \mathrm{Alq}_{3}$ interface. Interface charge density $\left(\sigma_{\text {int }}\right)$ was estimated to be ca. $-1.1 \mathrm{mC} / \mathrm{m}^{2}$. The interface charge defines the least amount of the accumulated charge in the operating device. This is because charge accumulation occurs at biases below $V_{\mathrm{bi}}$ and the actual current starts flowing after the compensation of interface charge by accumulated charge. Moreover, Kondakov et al. have reported that the apparent $\sigma_{\text {int }}$ decreases in proportion to the degradation of luminous efficiency. ${ }^{18}$ However, the origin of the interface charge still needs to be clarified.

Ito $e t$ al. reported the formation of giant surface potential (GSP) in an $\mathrm{Alq}_{3}$ film and its decay by light irradiation. ${ }^{19}$ The surface potential of an $\mathrm{Alq}_{3}$ film on a Au substrate shows linear growth with a slope of $50 \mathrm{mV} / \mathrm{nm}$ as the film thickness increases. The surface potential reaches $28 \mathrm{~V}$ at $560 \mathrm{~nm}$ but diminishes by light absorption of the $\mathrm{Alq}_{3}$ film. Complementary studies using optical second harmonic generation (SHG) revealed that GSP originates from the spontaneous order of the permanent dipole moment of $\mathrm{Alq}_{3} .{ }^{19,20}$ These results indicate that the $\mathrm{Alq}_{3}$ film has orientation polarization that induces a constant surface charge of $1.42 \mathrm{mC} / \mathrm{m}^{2}$ on the film independent of its thickness. This value is similar to $\sigma_{\text {int }}$ found at the $\alpha-\mathrm{NPD} / \mathrm{Alq}_{3}$ interface in OLEDs, but the polarity is opposite. However, the same amount of negative charge should exist at the bottom side of the $\mathrm{Alq}_{3}$ film, and this negative charge should appear at the $\alpha$-NPD/Alq ${ }_{3}$ interface in bilayer OLEDs, where $\mathrm{Alq}_{3}$ is deposited on the $\alpha$-NPD layer. Because of the light-induced decay property, it has been believed that GSP does not persist in the actual device. Furthermore, GSP has not been studied in terms of device properties; however, GSP can induce the interface charge, if the orientation polarization persists in the actual OLEDs.

GSP is not a unique property of $\mathrm{Alq}_{3}$ films. It has been found in films of several compounds such as metal complexes, ${ }^{21}$ 1,3,5-tris(1-phenyl-1 H-benzimidazol-2-yl)benzene (TPBi), ${ }^{22}$ and 2,9-dimethyl-4,7-diphenyl-1,10-phenanthroline (BCP). ${ }^{23}$ Furthermore, the presence of interface charge has also been suggested at the $\alpha$-NPD/1,3-bis[2-(4-tertbutylphenyl)-1,3,4-oxadiazo-5-yl]benzene (OXD-7) interface. ${ }^{17}$ Kondakov et al. reported voltammetry data for several devices in which charge injection voltage appears at the reverse biasing voltages, suggesting the presence of interface charge. ${ }^{24,25}$ These studies imply that interface charge and GSP can exist even in common OLEDs; however, a direct comparison between interface charge and GSP has not yet been demonstrated. Clarifying the role of polarization charge for the charge accumulation properties of a device is essential for a more accurate understanding and control of device properties.

In this study, we performed displacement current measurements (DCMs) for bilayer devices consisting of $\alpha$-NPD and several kinds of polar or nonpolar molecular layers, and Kelvin probe (KP) measurements for the corresponding bilayer structures for a quantitative comparison between interface charge density and GSP intensity. We found that films composed of polar molecules exhibit GSP behavior and interface charge appears in devices containing such films; however, neither GSP nor interface charge appears in devices with films composed of nonpolar molecules. These experimental results strongly suggest that orientation polarization induces interface charge in bilayer devices. In addition, we examined the charge behavior at the interface in bilayer devices using impedance spectroscopy (IS) and found that the presence of a permanent dipole moment in the vicinity of an organic heterointerface suppresses the charge spreading along the interface. This behavior can be explained by the microscopic potential fluctuation due to the dipole moment in the adjacent film. Our results clearly demonstrate that the permanent dipole moment of a molecule and its orientational order in the evaporated film are important factors for understanding and controlling the charge accumulation properties of a device.

\section{METHODS}

DCM is a type of capacitance-voltage $(C-V)$ measurement that uses a triangular wave form as the applied voltage and measures both actual and displacement current responses $\left(i_{\text {act }}\right.$ and $\left.i_{\text {dis }}\right)$. When the sweep rate of the applied triangular voltage $(d V / d t)$ is sufficiently slow compared to the transient response of charges in the device (at the quasi-static limit), the measured current $\left(i_{\mathrm{DCM}}\right)$ can be expressed as in ${ }^{10,26}$

$$
i_{\mathrm{DCM}}=i_{\text {act }}+C_{\text {app }} \frac{d V}{d t},
$$

where $C_{\text {app }}$ is the apparent capacitance of the device. $C_{\text {app }}$ directly provides information regarding the charge injection/ extraction and accumulation properties of a device. Figure 1(a) shows a schematic illustration of a typical DCM curve for a bilayer device; for simplicity, the second layer (the cathode side) is assumed to be an insulator. A DCM curve has three states; depletion, intermediate, and accumulation. In the depletion state, no charge carriers exist in the device, and thus, the observed $C_{\text {app }}$ corresponds to the total capacitance of the device [Fig. 1(b) (i)]. On the other hand, $C_{\text {app }}$ increases to the capacitance of the insulating layer in the accumulation state because the injected charges from the anode accumulate at the interface between the first and second layers, and then, only the second layer functions as a dielectric layer [Fig. 1(b) (iii)]. In addition, an intermediate state between the depletion and accumulation states often appears in DCM curves. The intermediate state suggests change in the depletion width in the 

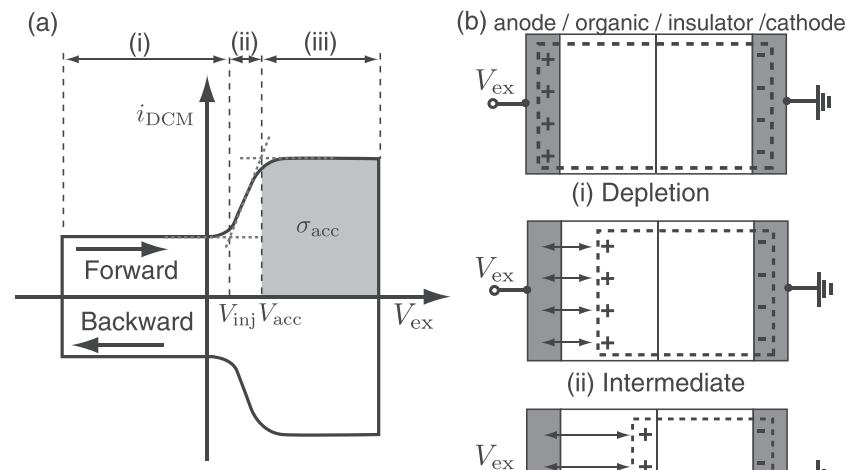

(i) Depletion

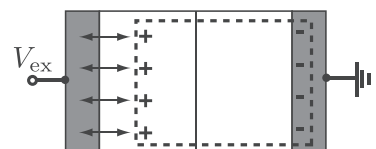

(ii) Intermediate

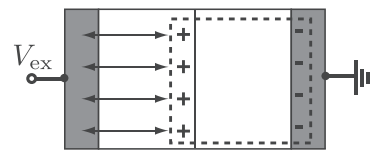

(iii) Accumulation

FIG. 1. (a) Schematic illustration of a typical DCM curve for a bilayer device. There are three states in the DCM curve: (i) depletion, (ii) intermediate, and (iii) accumulation. $V_{\text {inj }}$ and $V_{\text {acc }}$ are defined by the intersection of the extended lines from each state. The amount of accumulated charges at the interface is proportional to the gray area. (b) Schematic illustrations of charge location in the bilayer device in each state.

first layer [Fig. 1(b) (ii)], which can originate from the presence of intentional or unintentional dopants and the charge filling process of gap states. Here, we define the onset of charge injection from the electrode, $V_{\text {inj }}$, and accumulation at the interface, $V_{\text {acc }}$, as the intersection of the lines extending from each state, as shown in Fig. 1(a).

The interface charge density of bilayer devices can be evaluated by the DCM technique. ${ }^{27,28}$ If negative interface charge exists in a bilayer device, hole injection occurs at biases lower than the threshold voltage of the actual current $\left(V_{\text {th }}\right)$. In this bias range, the second layer acts as an insulator; in other words, no charges cross the interface. Therefore, the energy barrier and mobility mismatch at the interface are not responsible for the amount of accumulated charge. Hole injection starts at $V_{\text {inj }}$ and the injected holes penetrate into the first layer through the intermediate state. At $V_{\text {acc }}$, the injected holes reach the heterointerface and accumulate until the negative interface charge has been compensated. ${ }^{17}$ Thus, $\sigma_{\text {int }}$ can be obtained as

$$
-\sigma_{\mathrm{int}}(\mathrm{DCM})=\int_{V_{\mathrm{acc}}}^{V_{\mathrm{th}}} C_{\mathrm{app}} d V .
$$

Although quasi-static DCM is similar to conventional $C-V$ measurement, DCM detects both injected and extracted charge carriers and hence can obtain the amount of trapped charge as well as polarization switching in a device. This is because the true current (not the root mean square) is measured as a function of time and applied voltage. The details of this technique are described elsewhere. ${ }^{10,26}$

In addition, we measured the surface potential of the bilayer structure by the KP method to examine the correlations between GSP and interface charge. The observation of GSP strongly suggests the presence of orientation polarization in the film and $\sigma_{\text {int }}$ can also be evaluated from the thickness dependence of GSP. According to the principle of electric flux density continuity, the interface charge density between films 1 and 2 is given by

$$
\sigma_{\text {int }}(\mathrm{GSP})=\epsilon_{1} \frac{d}{d x} \Phi_{1}-\epsilon_{2} \frac{d}{d x} \Phi_{2}
$$

where $\epsilon_{n}$ and $\Phi_{n}(n=1,2)$ are the dielectric constant and surface potential of the film $n$, respectively, and the $x$ axis corresponds to the surface normal. Here, the real and polarization charges can be the origin of $\sigma_{\text {int }}$. Because GSP is proportional to film thickness, the product of the GSP slope and dielectric constant gives us interface charge density.

The charge carrier behavior at organic heterointerfaces was examined using IS and analyzed by $C-V$ and capacitance-frequency $(C-f)$ curves. ${ }^{29}$ In these measurements, an alternating voltage modulation $\left(V_{\mathrm{AC}} e^{j 2 \pi f t}\right)$ superimposed on a steady-state offset $\left(V_{\mathrm{DC}}\right)$ is applied to the device and the ac response including amplitude $\left(i_{\mathrm{AC}}\right)$ and phase shift $(\phi)$ are measured with respect to the applied ac modulation. Because the equivalent circuit of OLEDs can typically be described as a parallel circuit of resistance and capacitance, the capacitance at a given $V_{\mathrm{DC}}$ and $f$ is derived as

$$
C(f)=-\frac{1}{2 \pi f} \frac{Z^{\prime \prime}}{|Z|^{2}},
$$

where

$$
Z=Z_{0} e^{-j \phi}=Z^{\prime}+j Z^{\prime \prime} \text { and } Z_{0}=\frac{V_{\mathrm{AC}}}{i_{\mathrm{AC}}} .
$$

The $C-V(C-f)$ curves at a given $f\left(V_{\mathrm{DC}}\right)$ were measured by scanning $V_{\mathrm{DC}}(f)$.

\section{EXPERIMENTAL}

For the DCM and IS measurements, we fabricated bilayer devices that had the structure of indium tin oxide (ITO)/ $\alpha$-NPD/X/Al or Au [Figs. 2(a) and 2(b)], where $X$ indicates polar $\left(\mathrm{Alq}_{3}, \mathrm{TPBi}, \mathrm{BCP}, \mathrm{OXD}-7\right)$ or nonpolar molecules $[1,4-$ bis(triphenylsilyl)benzene (UGH-2), 4,4'-bis( $N$-carbazolyl)-1, $1^{\prime}$-biphenyl (CBP)]. The structure and basic properties of these molecules are summarized in Fig. 3 and Table I, respectively. The films in each device were successively formed through a conventional evaporation technique under a high vacuum condition at a base pressure of $\sim 10^{-4} \mathrm{~Pa}$. Note that samples were fabricated in the dark without ion gauge operation throughout the film deposition processes, because ambient light and ion gauge operation can change the electronic properties of the resultant device. ${ }^{30}$ The samples were directly transferred to a glove box filled with nitrogen, and the electronic properties were measured in the glove box or a vacuum chamber. The measurement setups are shown in Figs. 2(a) and 2 (b).

For the DCM measurements, we employed the FCE-1 measurement system for ferroelectrics (Toyo Corp.). All DCM curves shown in this paper were measured at a sufficiently low sweep rate $(1 \mathrm{~V} / \mathrm{s})$ at room temperature. The surface potential of the films was measured under a high vacuum condition by the KP method (McAllister KP6500) using the work function of the ITO substrate as reference. The films were successively deposited on the ITO substrate and the surface potential was measured in situ at each step of the deposition. The 
(a) Top View
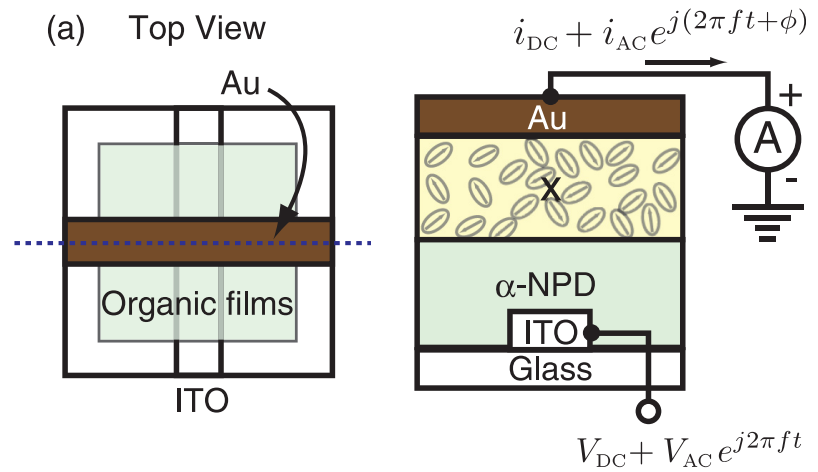

(b) Top View
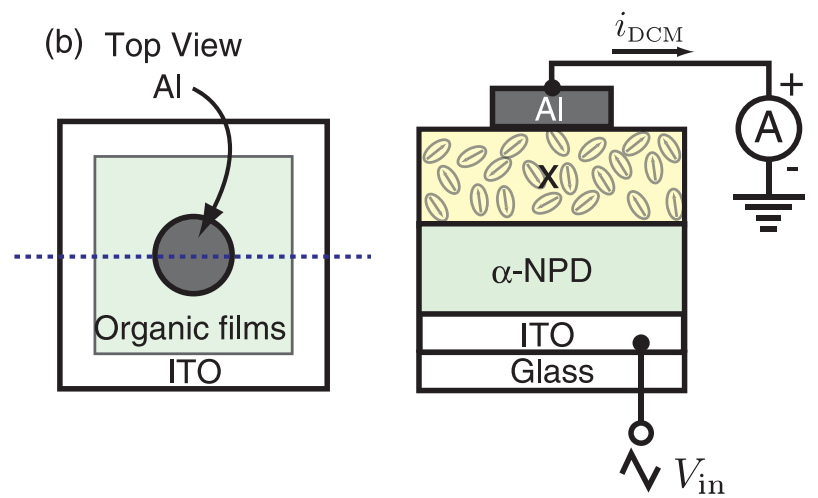

(c)

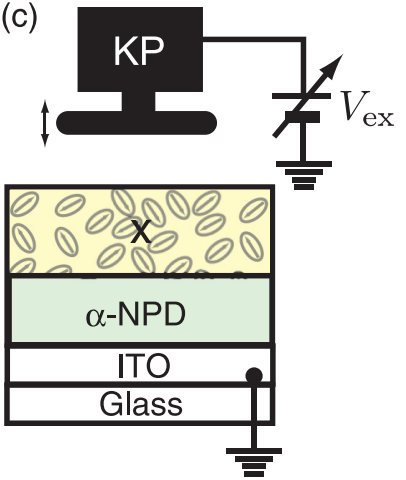

FIG. 2. Schematic illustration of the sample structure and experimental setup for (a) $C-V$ and $C-f$, (b) DCM, and (c) KP measurements. For $C-V$ and $C-f$ measurements, a cross-bar structure was employed. The width of the top and bottom electrodes was $2 \mathrm{~mm}$ and the area of the organic films was $12 \times 12$ $\mathrm{mm}^{2}$. For DCM, an island-type top electrode with a diameter of $1.5 \mathrm{~mm}$ was used. The broken lines in (a) and (b) indicate where the cross-section for the profiles was taken. Tip of KP with a diameter of $5 \mathrm{~mm}$ was located about $0.5 \mathrm{~mm}$ above the film surface.

experiment was performed in the dark without ion gauge operation to avoid altering the surface potential. The $C-V$ and $C-f$ curves were measured using a frequency response analyzer (Solartron SI 1260 impedance/gain-phase analyzer) combined with a dielectric interface (Solartron 1296) at temperatures of $200-350 \mathrm{~K}$. For the $C-V$ measurement, $V_{\mathrm{DC}}$ was swept at fixed frequencies of $1-10^{5} \mathrm{~Hz}$, whereas for the $C-f$ measurement, $f$ was varied from $10^{-2}$ to $10^{7} \mathrm{~Hz}$ at a given $V_{\mathrm{DC}} . V_{\mathrm{AC}}$ was set to $0.1 \mathrm{~V}$ for all measurements.

\section{RESULTS AND DISCUSSION}

\section{A. Interface charge and orientation polarization}

Figure 4 shows the typical surface potential of $\alpha-\mathrm{NPD}$ and $\mathrm{Alq}_{3}$ films as a function of film thickness. A clear GSP

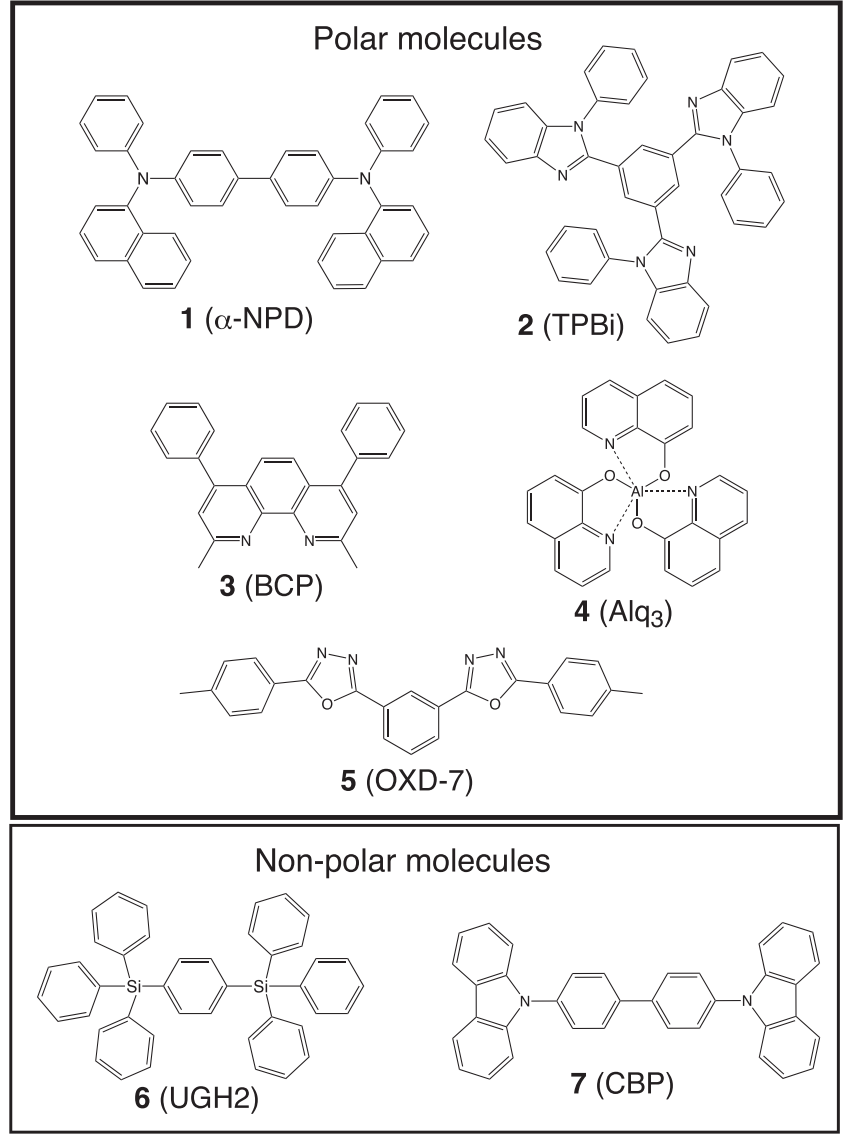

FIG. 3. Chemical structures of the molecules used in this study. Here, 1-5 are polar, whereas 6 and 7 are nonpolar molecules.

behavior with a slope of $48 \mathrm{mV} / \mathrm{nm}$ is observed for the $\mathrm{Alq}_{3}$ film, indicating that the film has orientation polarization. The corresponding surface charge density is $1.36 \mathrm{mC} / \mathrm{m}^{2}$ with a relative dielectric constant 3.2 estimated from capacitance measurement. A potential jump of about $0.2 \mathrm{eV}$ is observed at the interface region ( $\Delta$ in the bottom inset); this jump implies the presence of an interface dipole that results in a vacuum level shift. ${ }^{31}$ Interestingly, the $\alpha$-NPD film also shows weak GSP behavior $(\sim 5.3 \mathrm{mV} / \mathrm{nm}$, top inset); thus, the total $\sigma_{\text {int }}$ of the $\alpha-\mathrm{NPD} / \mathrm{Alq}_{3}$ interface can be estimated as $-1.2 \mathrm{mC} / \mathrm{m}^{2}$ [Eq. (3)]. GSP behavior also appeared in the films composed of the other polar molecules including OXD-7, BCP, and TPBi (Fig. 5). The largest GSP was observed in the OXD-7 film, i.e., $68 \mathrm{mV} / \mathrm{nm}$ corresponding to $-1.6 \mathrm{mC} / \mathrm{m}^{2}$ of $\sigma_{\text {int }}(\mathrm{GSP})$. On the other hand, the surface potential of the films comprising the nonpolar molecules, UGH2 and CBP, does not show GSP, although it weakly depends on the film thickness probably owing to charge transfer from $\alpha$-NPD. The results are summarized in Table I.

Figure 6(a) shows a typical DCM curve for the bilayer device, in which an $\mathrm{Alq}_{3}$ film was used as the second layer. According to the observed DCM curve, the carrier behavior of this device can be explained as follows. The triangular bias sweep starts at $-5 \mathrm{~V}$. Around the start voltage, current density is maintained at a lower level $\left(\sim 0.026 \mu \mathrm{A} / \mathrm{cm}^{2}\right) . C_{\text {app }}$ corresponds to the total capacitance of the device in this bias region, indicating that the device is in the depletion state [(i) in Fig. 6(b)]. Subsequently, the current increases at a voltage 
TABLE I. Interface properties of OLED-related materials. The dipole moment ( $p$ ) was calculated by GAUSSIAN 03 with a basis set of B3LYP/6-31 G(d). $\Delta E_{\mathrm{UPS}}$ is the highest occupied molecular orbital energy offset directly measured through ultraviolet photoelectron spectroscopy (UPS). ${ }^{39}$ The films were deposited on the $\alpha$-NPD film unless explicitly stated. The relative dielectric constant $\left(\epsilon_{\mathrm{r}}\right)$ was determined from the capacitance measurement. $\sigma_{\text {int }}$ (GSP) is derived from Eq. (3) and $\sigma_{\text {int }}$ (DCM) is estimated by integrating DCM curves [Eq. (2)]. ( $\ddagger$ : Ref. $40, \dagger: \alpha$-NPD on Alq ${ }_{3},{ }^{31} *$ : on ITO, $* *$ : The surface potential slightly depends on the film thickness (but not proportionally) probably owing to the charge transfer; however, the slope was estimated from the best-fit line in the same manner as for the other films./: not applicable).

\begin{tabular}{lcccccc}
\hline \hline & TPBi & BCP & Alq $_{3}$ & OXD-7 & CBP & UGH2 \\
\hline$p(\mathrm{D})$ & 2.0 & 2.9 & 4.4 & 6.5 & 0 & 0 \\
$\epsilon_{\mathrm{r}}$ & 2.87 & 3.45 & 3.2 & 2.9 & $2.7_{2}$ & 0.6 \\
$\Delta E_{\text {UPS }}(\mathrm{eV})$ & $1.1_{5}$ & $1.0^{\dagger}$ & $0.3^{\dagger}$ & 1.3 & 0.6 & $2.8_{7}$ \\
GSP slope $(\mathrm{KP})(\mathrm{mV} / \mathrm{nm})$ & 43 & 33 & 48 & 68 & $0.7^{* *}$ & $-2^{* *}$ \\
GSP slope $(\mathrm{DCM})(\mathrm{mV} / \mathrm{nm})$ & 37 & 12 & 34 & 87 & -6.5 & -6.2 \\
$\sigma_{\text {int }}(\mathrm{GSP})\left(\mathrm{mC} / \mathrm{m}^{2}\right)$ & -0.93 & -0.84 & -1.2 & -1.6 & 0.74 & $0.21^{*}$ \\
$\sigma_{\text {int }}(\mathrm{DCM})\left(\mathrm{mC} / \mathrm{m}^{2}\right)$ & -1.1 & -0.51 & -1.1 & -2.3 & 0 & 0.16 \\
\hline
\end{tabular}

of $-1.25 \mathrm{~V}\left(V_{\text {inj }}\right)$ owing to hole injection from the ITO substrate. The current increases until the biasing voltage reaches $-0.46 \mathrm{~V}\left(V_{\text {acc }}\right)$. At $V_{\text {acc }}$, the $\alpha$-NPD layer is expected to be in the flat-band condition, and thus, the potential due to interface charge builds up in the second layer [(ii) in Fig. 6(b)]. The current again shows constant intensity at voltages lower than $1.9 \mathrm{~V}\left(V_{\text {th }}\right) . C_{\text {app }}$ in this bias region corresponds to the capacitance of the $\mathrm{Alq}_{3}$ layer, indicating that hole accumulation occurs at the $\alpha-\mathrm{NPD} / \mathrm{Alq}_{3}$ interface. $V_{\text {th }}$ corresponds to the threshold voltage of the actual current and it agrees well with $V_{\mathrm{bi}}$ of the device (typically $\sim 1.9 \mathrm{~V}$ ) estimated from the open-circuit voltage during intense white light irradiation. This indicates that when the accumulated holes compensate interface charge, the flat-band condition is achieved throughout the device and the actual current flows through the device [(iii) in Fig. 6(b)]. $\sigma_{\text {int }}$ is estimated from the DCM curve to be $-1.1 \mathrm{mC} / \mathrm{m}^{2}$ [Eq. (2)].

Figures 7(a) and 7(b) show the DCM curves for bilayer devices of $\mathrm{Alq}_{3}$ containing various combinations of film thicknesses. The current density at the accumulation state is

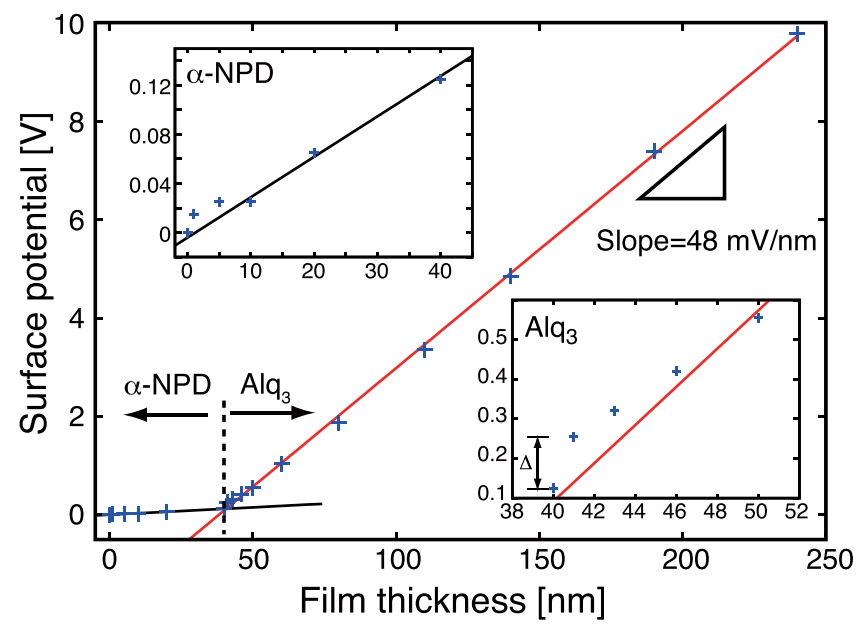

FIG. 4. The surface potential of the $\mathrm{Alq}_{3}$ and $\alpha$-NPD films as a function of film thickness. The $\mathrm{Alq}_{3}$ film was deposited on an $\alpha$-NPD film on an ITO substrate. The $\alpha-\mathrm{NPD} / \mathrm{Alq}_{3}$ interface is located at a film thickness of $40 \mathrm{~nm}$. The surface potential of the $\mathrm{Alq}_{3}$ film grows linearly with a slope of $48 \mathrm{mV} / \mathrm{nm}$, although a nonlinear region appears within several nanometers from the interface (bottom inset). The potential jump at the $\mathrm{Alq}_{3} / \alpha$-NPD interface $(\Delta)$ suggests the presence of an interface dipole. The $\alpha$-NPD film also shows weak GSP behavior with a slope of ca. $5.3 \mathrm{mV} / \mathrm{nm}$ (top inset). higher for the devices with a thinner $\mathrm{Alq}_{3}$ layer [Fig. 7(a)], and it is independent of the $\alpha$-NPD film thickness [Fig. 7(b)]. These results indicate that the $\mathrm{Alq}_{3}$ layer acts as an insulator at the accumulation state, which shows that the charges injected from the ITO substrate are holes. In addition, $V_{\text {th }}$ is independent of the film thicknesses in all devices, whereas $V_{\text {acc }}$ shifts to the negative side with increasing $\mathrm{Alq}_{3}$ film thickness, as previously reported. ${ }^{17,32}$ On the other hand, $V_{\text {acc }}$ is independent of the $\alpha$-NPD film thickness. Here, $V_{\text {acc }}$ can be described as

$$
V_{\mathrm{acc}}=V_{\mathrm{th}}+\frac{\sigma_{\mathrm{int}}}{\epsilon_{\mathrm{Alq}}} d_{\mathrm{Alq}},
$$

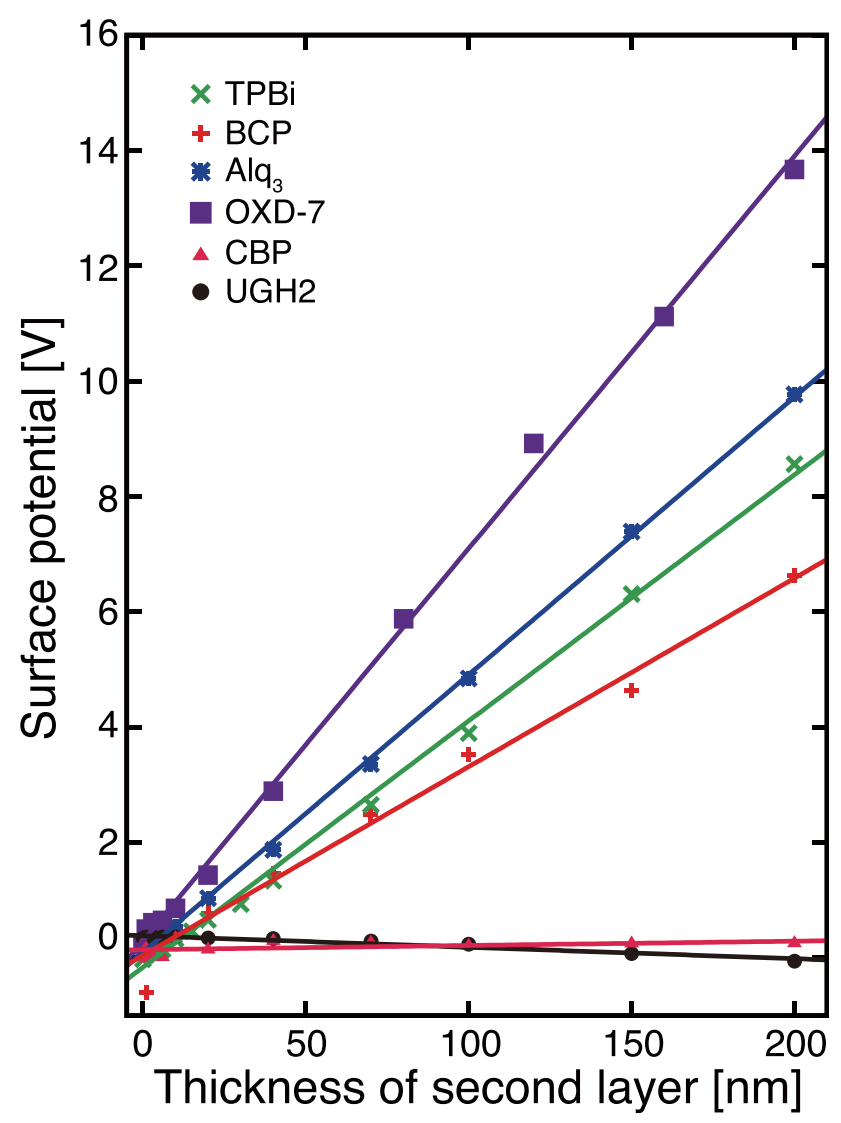

FIG. 5. Surface potential of various films on an $\alpha$-NPD layer. 

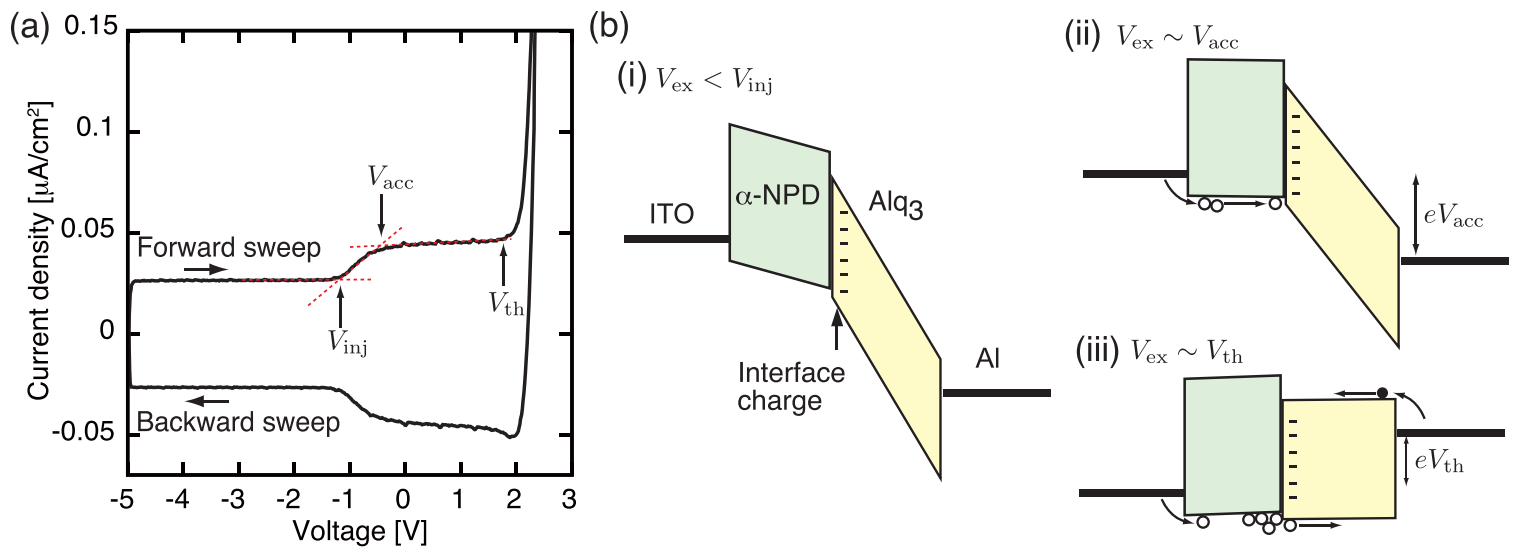

FIG. 6. (a) A typical DCM curve for the ITO/ $\alpha-\mathrm{NPD} / \mathrm{Alq}_{3} / \mathrm{Al}$ device at a sweep rate of $1 \mathrm{~V} / \mathrm{s}$. (b) Schematic illustrations of an energy diagram at each bias range.

where $\epsilon_{\mathrm{Alq}}$ and $d_{\mathrm{Alq}}$ are the dielectric constant and thickness of the $\mathrm{Alq}_{3}$ film, respectively. The slope of the $V_{\text {th }}-V_{\text {acc }}$ vs. $d_{\text {Alq }}$ plot (Fig. 8) gives us $40 \mathrm{mV} / \mathrm{nm}\left(=-\sigma_{\text {int }} / \epsilon_{\text {Alq }}\right)$ corresponding to the electric field built up in the $\mathrm{Alq}_{3}$ layer at $V_{\text {acc }}$. This value includes the contribution from GSP of the $\alpha$-NPD layer; however, using Eq. (3), the GSP slope of the $\mathrm{Alq}_{3}$ layer can be obtained as

$$
\frac{d \Phi_{\mathrm{Alq}}}{d x}=\frac{d}{d x}\left(V_{\mathrm{th}}-V_{\mathrm{acc}}\right)-\frac{\epsilon_{\mathrm{NPD}}}{\epsilon_{\mathrm{Alq}}} \frac{d}{d x} \Phi_{\mathrm{NPD}} .
$$

The GSP slope of the $\mathrm{Alq}_{3}$ layer is then estimated to be $34 \mathrm{mV} / \mathrm{nm}$; this value is similar to that obtained from the KP measurement. The GSP slope values of the $\alpha$-NPD film and the relative dielectric constants used for the estimation are shown in Table I. Similar characteristics were observed in the DCM curves for all bilayer devices containing GSP films, i.e., films exhibiting GSP behavior; OXD-7, BCP, and TPBi (Fig. 8). The linear dependence on film thickness indicates that GSP films induce a constant interface charge density independent of film thickness.

Figure 9(a) shows a typical DCM curve for a bilayer device with a nonpolar film as the second layer (ITO/ $\alpha$-NPD/ $\mathrm{UGH} 2 / \mathrm{Al}$ device). $V_{\text {acc }}$ appears at about $1.6 \mathrm{~V}$, but $V_{\text {th }}$ is unclear. Therefore, we derived $i_{\text {act }}$ and $i_{\text {dis }}$ from $\left(i_{+}+i_{-}\right) / 2$ and $\left(i_{+}-i_{-}\right) / 2$, respectively. Here, $i_{+}$is the DCM current observed in the forward sweep $(d V / d t>0)$ and $i_{-}$is that observed in the backward sweep $(d V / d t<0)$. Because $i_{\text {dis }}$ divided by the sweep rate corresponds to $C_{\text {app }}$, the $C_{\text {app }}-V$ curve is finally obtained. Figure 9(b) shows the extracted $C_{\text {app }}-V$ and $i_{\text {act }}-V$ curves, where both $V_{\text {acc }}$ and $V_{\text {th }}$ are observed at $1.6 \mathrm{~V}$, indicating no interface charge in the devices. We confirmed that for the CBP device, $V_{\text {acc }}$ corresponds to $V_{\text {th }}$.

Figure 10 compares the KP and DCM results. Figure 10(a) plots the GSP slope in the second layer determined from the KP and DCM data. Here, the GSP slope obtained from DCM was estimated from Eq. (6). Figure 10(b) shows the relationship between $\sigma_{\text {int }}(\mathrm{GSP})$ and $\sigma_{\text {int }}$ (DCM). $\sigma_{\text {int }}$ (GSP) was determined by Eq. (3). The parameters shown in Table I were used for the calculations. As shown in these figures, $\sigma_{\text {int }}$ and GSP obtained from two different experiments seem to correlate well, and the data points are distributed around the line with a slope of 1 . Thus, we conclude that interface charge and GSP can both be attributed to orientation polarization in the film. However, a quantitative disagreement still remains between the results of the KP measurement and DCM. For instance, the OXD-7 film shows a smaller GSP slope than that estimated from the DCM result. A possible reason for this disagreement is the underestimation of the orientation polarization in the KP measurement. Surface potential, especially GSP, is sensitive to temperature, ambient light, and substrate properties, rather than the interface charge observed in the
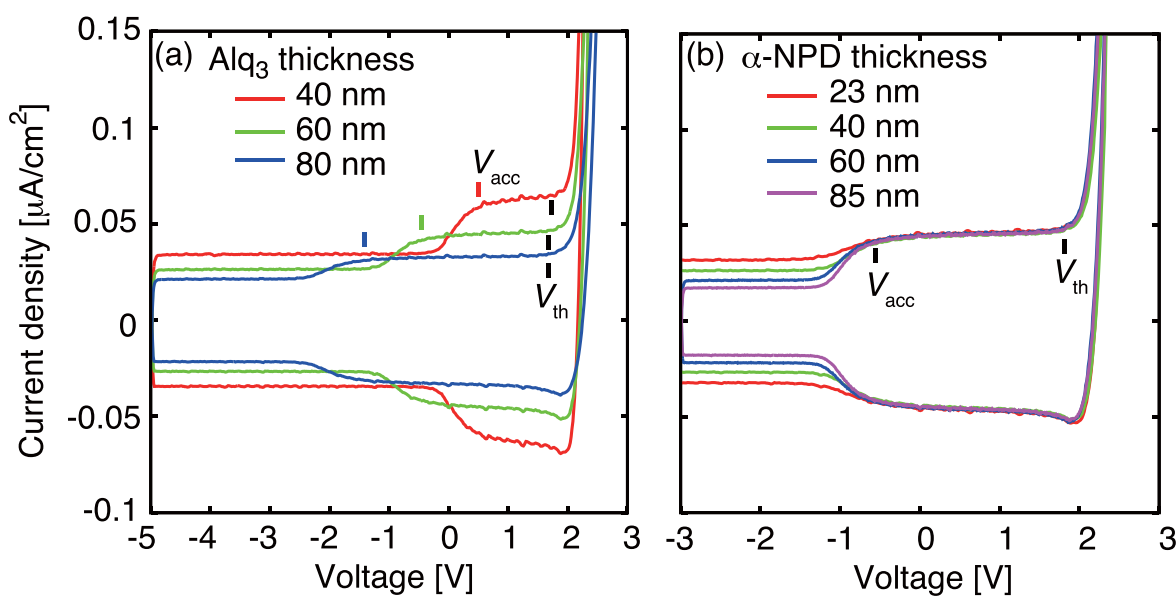

FIG. 7. (a) and (b) DCM curves of the ITO/ $\alpha$-NPD/Alq $/ 3 / \mathrm{Al}$ device for various film thicknesses. The $\alpha$-NPD and $\mathrm{Alq}_{3}$ film thicknesses are fixed at $40 \mathrm{~nm}$ (a) and $60 \mathrm{~nm}$ (b), respectively. The current density at the accumulation state depends only on the $\mathrm{Alq}_{3}$ film thickness, indicating that the injected charges are holes. The threshold voltage of the actual current $V_{\text {th }}$ is independent of the combinations of the film thickness, whereas $V_{\text {acc }}$ shifts to the negative side with increasing $\mathrm{Alq}_{3}$ film thickness. Thus, the polarity of interface charge is negative. 


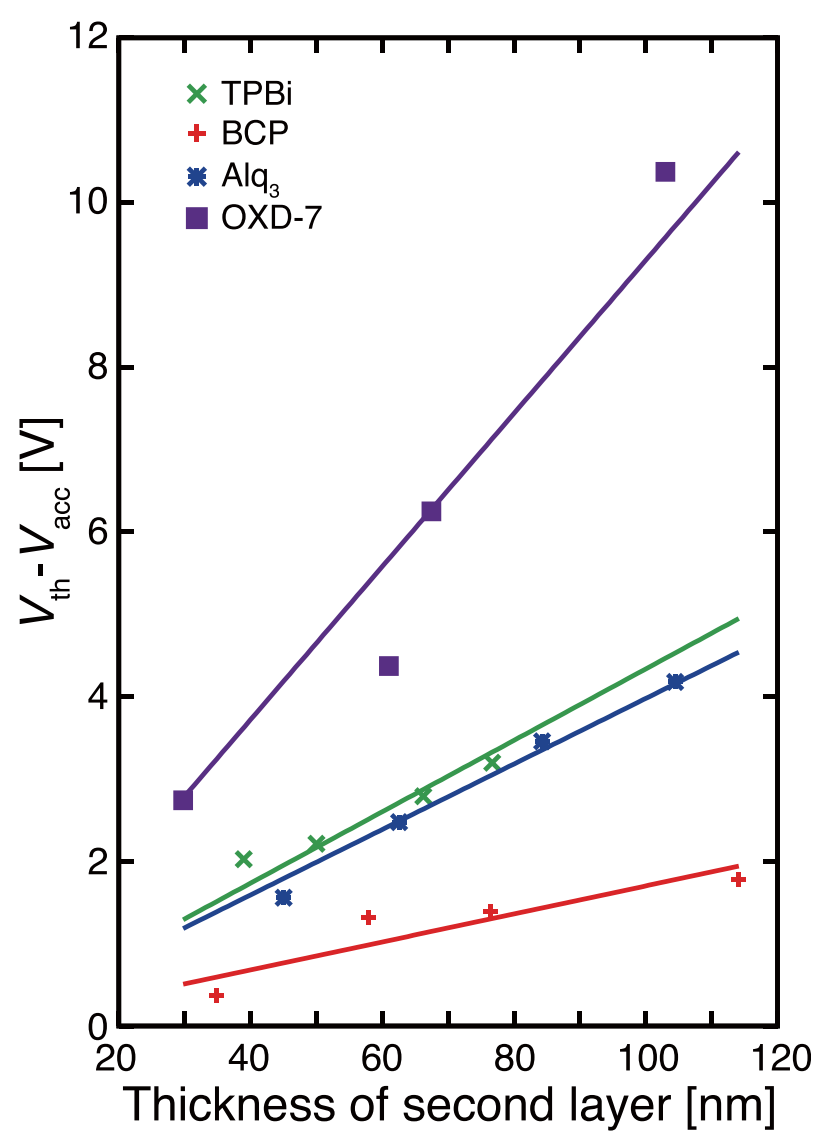

FIG. 8. Thickness dependence of $V_{\text {th }}-V_{\text {acc }}$ determined from the DCM curves for the bilayer devices.

device (as discussed later). This is because GSP decays irreversibly owing to the presence of compensation charges originating from the electrode (as thermally injected carriers), the bulk of the film (as photoinduced carriers), and the environment. ${ }^{19,33}$ Thus, intrinsic orientation polarization can be larger than that estimated from GSP. In addition, the detailed conditions of the film deposition procedure may also affect orientation polarization in the resultant film. In the KP experiment, film deposition was interrupted for surface potential measurement at specific film thicknesses, whereas it was performed continuously for device fabrication. Although further analyses are required to quantitatively understand these results, they strongly suggest that GSP and interface charge originate from the orientation polarization and can be produced in common organic semiconductor films with polar molecules.
GSP of $\mathrm{Alq}_{3}$ films and its decay have been mainly investigated by the KP and SHG techniques. ${ }^{19,20,33-36}$ For the photoinduced decay of GSP, two possible mechanisms have been proposed; one is the photoinduced disorder of the dipole moments and the other is compensation of the polarization charge on the surface by the counter charge of photogenerated carriers in the film. Previous studies have not sufficiently clarified the mechanism, mainly owing to the experimental difficulties of this system, for example, the small order parameter even at the initial state (typically $\langle\cos \theta\rangle \sim 0.05$, indicating almost random but slightly ordered on average, where $\theta$ is the tilt angle of the dipole moment with respect to the surface normal) and the irreversible decay of GSP caused by light irradiation and thermal fluctuations. However, our experimental results strongly support the conclusion that the generation of compensation charge is the origin of the photoinduced decay of GSP. If the photoinduced disorder is the dominant mechanism, the film loses orientation polarization and interface charge in the bilayer device should disappear. In the case of compensation charge, orientation polarization and interface charge remains in the device and the counter charge can be removed by applying an external electric field. We have confirmed that the interface charge density persists even after intense white light irradiation. Thus, we expect that the DCM technique can evaluate the intrinsic interface charge density, and this technique can be a powerful tool to investigate the orientation polarization properties of organic semiconductor films.

The mechanisms of spontaneous ordering in the evaporated film of polar molecules still need to be clarified; however, the correlations between Coulomb interactions and the molecular packing should be responsible for the structure of the resultant film. ${ }^{11}$ Here, as the simplest case, we consider the interaction between two identical molecules with a permanent dipole moment of $\mathbf{p}$ in a parallel configuration. The potential energy of these two dipoles $\left(\mathcal{V}_{\uparrow \uparrow}\right)$ is given by ${ }^{37}$

$$
\mathcal{V}_{\uparrow \uparrow}=-\frac{1}{4 \pi \epsilon_{0}} \frac{p^{2}}{r^{3}}\left(3 \cos ^{2} \beta-1\right),
$$

where $r$ is the distance between two molecules and $\cos \beta=\mathbf{p} \cdot \hat{\mathbf{r}} /|\mathbf{p}| . \hat{\mathbf{r}}$ is the unit vector pointing from a reference molecule to the other molecule. Similarly, the potential of the antiparallel configuration can be obtained as $\mathcal{V}_{\uparrow \downarrow}=-\mathcal{V}_{\uparrow \uparrow}$. For $\mathrm{Alq}_{3}$, using $p=4.4 \mathrm{D}\left(=1.47 \times 10^{-29} \mathrm{Cm}\right)$ and $r=8 \AA$,

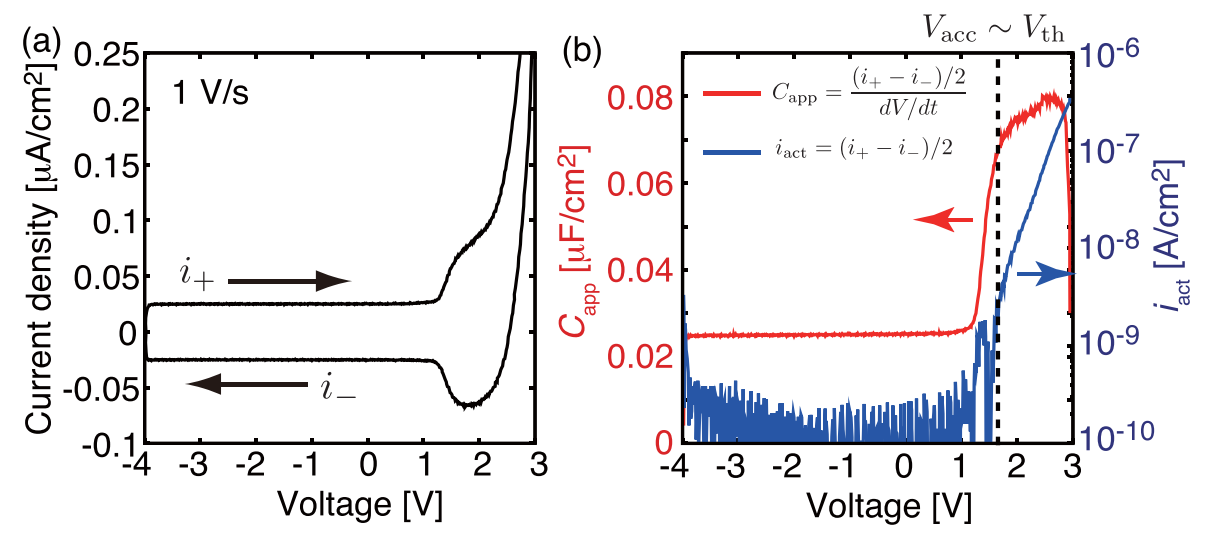

FIG. 9. (a) Typical DCM curve for the ITO/ $\alpha$-NPD/UGH2/Al device at a sweep rate of $1 \mathrm{~V} / \mathrm{s}$. (b) Capacitance-voltage and actual currentvoltage curves derived from the DCM curve. 

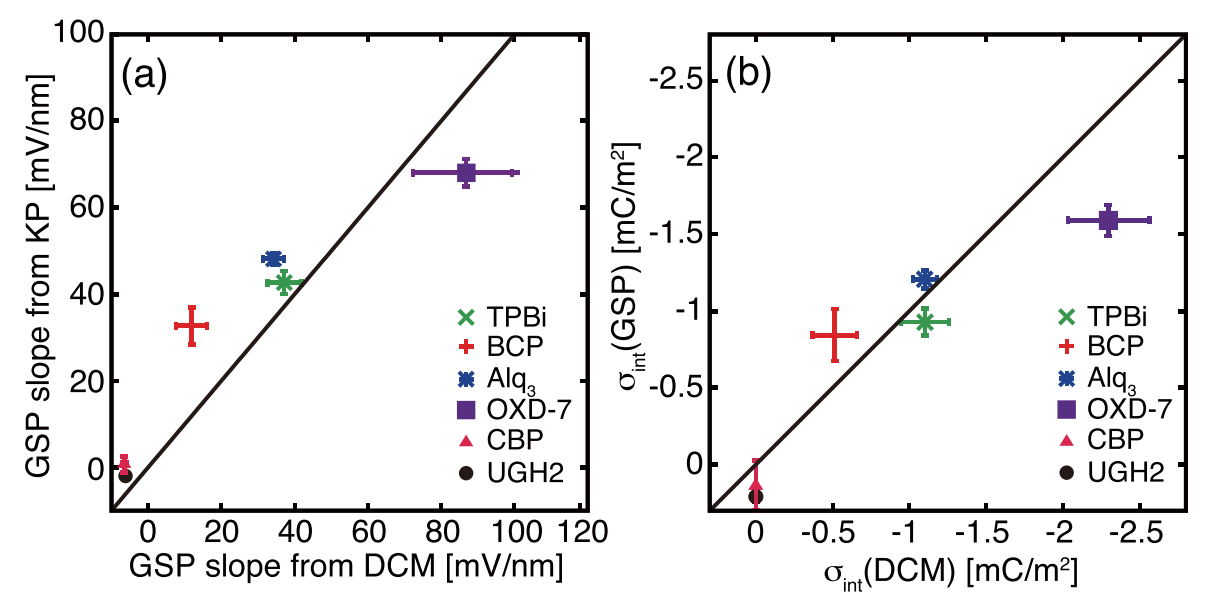

FIG. 10. Comparison between the KP and DCM results. (a) The electric field formed in the second layer determined by the KP measurement and DCM. (b) Interface charge density estimated from the GSP slope and DCM curves.
$-47.2 \mathrm{meV} \leq \mathcal{V}_{\uparrow \uparrow}\left(=-\mathcal{V}_{\uparrow \downarrow}\right) \leq 23.6 \mathrm{meV}$ is obtained. Here, $r$ is estimated from the $\mathrm{Alq}_{3}$ film density $\left(1.51 \mathrm{~g} / \mathrm{cm}^{3}\right)$. It should be noted that the parallel configuration takes lower energy on average compared with the antiparallel configuration. On the other hand, the thermal energy and the potential energy of these two configurations are comparable. This implies that the molecular dipoles can form mostly random but slightly ordered configuration in the film phase. Here, we define the molecular dependent term $\frac{p^{2}}{r^{3}}=m$. For $\mathrm{Alq}_{3}, m$ can be estimated as $4.2 \times 10^{-31} \mathrm{C}^{2} / \mathrm{m}$. For the other polar molecules used in this study, appropriate estimation of apparent $m$ value is not easy because of their anisotropic molecular structures unlike $\mathrm{Alq}_{3}$. For example, if we calculate $m$ of OXD-7 by assuming inter-molecular distance simply from its molecular size, $m$ varies from $10^{-32}$ to $10^{-30} \mathrm{C}^{2} / \mathrm{m}$ depending on the combinations of molecular direction. Although the accurate estimation of film structure is necessary to obtain the $m$ value, it can be expected to have a similar property (e.g., GSP and interface charge), if apparent $m$ of the material is $\sim 10^{-31} \mathrm{C}^{2} / \mathrm{m}$.

\section{B. Charge spreading along the interface}

Figure 11(a) shows typical $C-V$ curves for bilayer devices with BCP and UGH2 films used as the second layer. Because the top electrode of these devices was $\mathrm{Au}$, they operated as hole-only devices. At the $\alpha$-NPD/BCP interface, $V_{\text {acc }}$ appears at a negative bias of $-0.2 \mathrm{~V}$ owing to the presence of negative interface charge, and then, the capacitance increases to a constant value corresponding to hole accumulation. The
$C-V$ curve for the UGH2 device shows different behavior. Because the GSP does not build up in the UGH2 film, there is no interface charge in the device and $V_{\text {acc }}$ appears at a positive bias of $1.8 \mathrm{~V}$. Then, capacitance increases and remains constant for a short voltage range, $1.8-2.2 \mathrm{~V}$, but gradually increases again at higher applied voltages. Similar behavior was observed in the $C$ - $V$ curve for the CBP device, but not in the devices containing polar molecules, e.g., $\mathrm{BCP}, \mathrm{Alq}_{3}$, and OXD-7.

Figure 11(b) shows the $C-f$ curves for the BCP and $\mathrm{UGH} 2$ devices at $V_{\mathrm{DC}}$ before and after hole injection. The capacitance drop at frequencies higher than $10^{6} \mathrm{~Hz}$ is an artifact. In this frequency region, the signal from the test device is hidden by a parasitic impedance originating from the measurement system. Two constant capacitances appear in the $C-f$ curve for the BCP device at a $V_{\mathrm{DC}}$ of $2 \mathrm{~V}$. The high $(1.7 \mathrm{nF})$ and low $(0.8 \mathrm{nF})$ capacitances correspond to those of the BCP layer and the entire device with an active area of 4 $\mathrm{mm}^{2}$, i.e., the cross-bar area, respectively. Because the holes in the device cannot respond to the applied ac modulation at high frequencies, the entire device acts as a capacitor. At frequencies of about $10^{\circ}-10^{4} \mathrm{~Hz}$, the hole movement in the $\alpha$ NPD layer can sufficiently follow the ac modulation and only the BCP layer remains as the dielectric layer. Therefore, the transition frequency, where the capacitance changes from a low state to a high state, depends on the conductance of the $\alpha$-NPD film. On the other hand, the capacitance of the UGH2 device at the accumulation region $\left(V_{\mathrm{DC}}=2 \mathrm{~V}\right)$ increases rapidly at low frequencies $\left(<10^{2} \mathrm{~Hz}\right)$. The observed capacitance
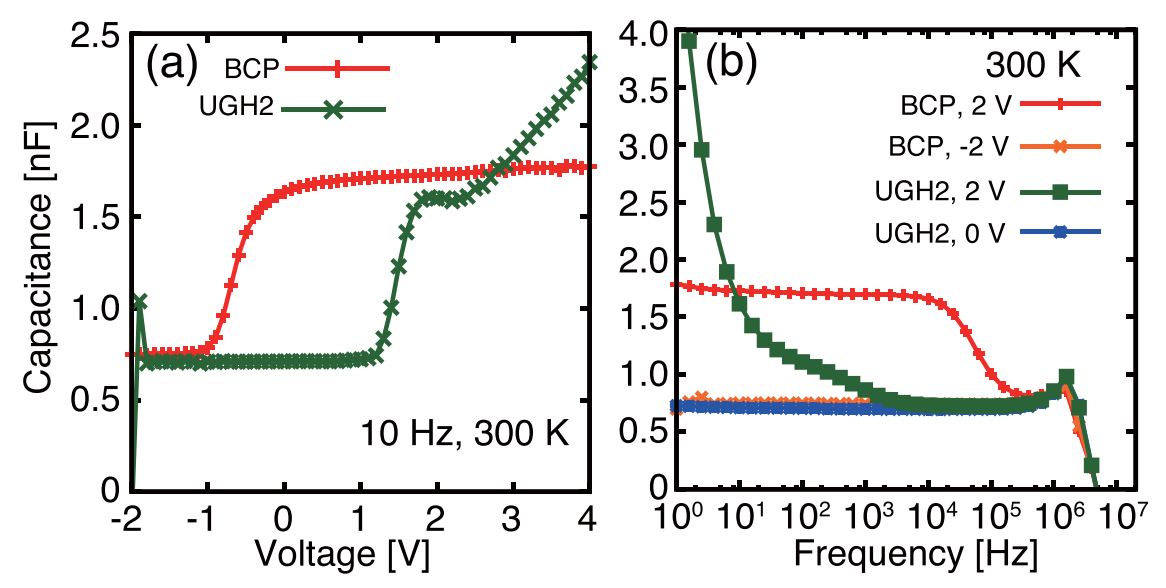

FIG. 11. (a) Typical $C-V$ curve for the BCP and UGH2 devices at $10 \mathrm{~Hz}$ and $300 \mathrm{~K}$. (b) Typical $C-f$ curves for the BCP and UGH2 devices. $V_{\mathrm{DC}}$ was -2 and $2 \mathrm{~V}$ for the $\mathrm{BCP}$ device and 0 and $2 \mathrm{~V}$ for the UGH2 device (corresponding to before and after hole injection, respectively). The measurement temperature was $300 \mathrm{~K}$ for both devices. 
(a)

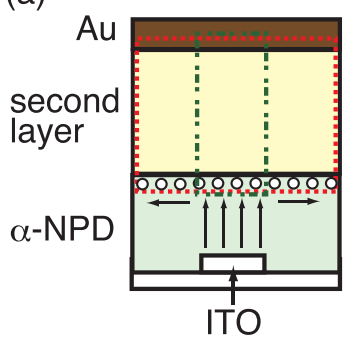

(b)

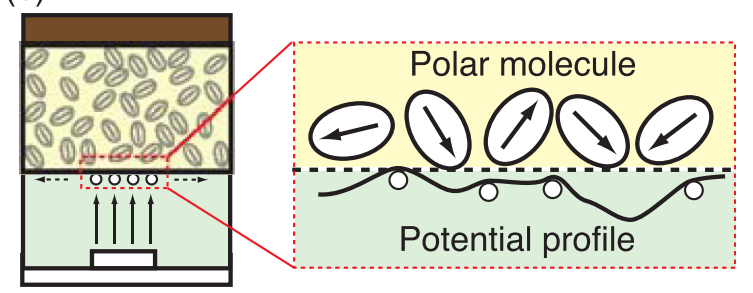

FIG. 12. (a) Schematic illustration of the bilayer device with charge spreading along the interface. The broken line (red) indicates the expanded area of capacitance, whereas the inner line (green) shows the cross-bar area. (b) Schematic illustration of the bilayer device in which the polar molecule is used as the second layer. The charge dispersion along the interface is suppressed owing to the microscopic potential fluctuation caused by the dipole moment. is much higher than that expected from the device geometry. This capacitance inflation also occurred in the BCP device at high temperatures (not shown), though the observed frequency is much lower than that in the UGH2 device. In addition, we measured the $C-V$ and $C-f$ curves for the bilayer devices containing $\mathrm{CBP}, \mathrm{Alq}_{3}$, and $\mathrm{OXD}-7$ films and found that the capacitance inflation in the nonpolar devices (CBP, $\mathrm{UGH} 2$ ) appeared at higher frequencies than that in the polar devices (BCP, $\mathrm{Alq}_{3}$, OXD-7).

Capacitance inflation is likely to occur in bilayer devices with nonpolar molecules. As shown in Fig. 11(b), the $C-f$ curve at a bias below the hole injection voltage $(-2 \mathrm{~V}$ for the $\mathrm{BCP}$ device and $0 \mathrm{~V}$ for the $\mathrm{UGH} 2$ device) is almost independent of frequency, indicating that hole injection is necessary for capacitance inflation. Thus, we can eliminate dielectric dispersion as the origin of this phenomenon. Here, two other possibilities should be considered; one is the decrease in the effective thickness of the dielectric layer, and the other is the expansion of the area that acts as a capacitor. The former case requires the presence of thermally activated or injected mobile charges in the bulk region of the second layer. If thermally activated carriers from the unintended dopant existed in our devices, capacitance change should be observed independent of hole injection. Moreover, from the KP measurements, the UGH2 and CBP films do not show a significant band bending even at the interface, suggesting that there are no thermally activated carriers in the films. On the other hand, a very large energy barrier is formed at the $\alpha$-NPD/UGH2 interface ( $\sim 2 \mathrm{eV}$, Table I). Because the height of this energy barrier is the largest among the interfaces used in this study, compared to the other interfaces, hole injection beyond this interface is unlikely to occur. Accordingly, the decrease in the dielectric thickness of the second layer also cannot explain our experimental results.

Because our device structure was a cross-bar type in which the deposition area of the organic films $\left(144 \mathrm{~mm}^{2}\right)$ is much larger than the cross-bar area $\left(4 \mathrm{~mm}^{2}\right)$, there is enough space for the accumulated charge carriers to spread along the organic heterointerface. The spreading of the charge carrier makes the interface conductive, and as a result, large capacitance formed between the carrier sheet and top stripe electrode is observed [Fig. 12(a)]. Because the amount of accumulation charge at the interface increases with applied voltage, higher applied voltage enhances the interface conductivity. Therefore, the capacitance grows with applied voltage as shown in the $C-V$ curve of the UGH2 device [Fig. 11(a)]. On the other hand, capacitance inflation is not significant in the DCM curve for the UGH2 device [Fig. 9(b)]. In this device, an island-type top electrode was used. The capacitance in the accumulation state is determined by the top electrode area, regardless of the spreading of the accumulated holes at the interface. According to the above discussion, the results of the $C-V$ and $C-f$ curves suggest that the conductance of holes along the heterointerface in the nonpolar devices is higher than that in the polar devices, although intrinsic conductance should be the same in all bilayer devices. This is because the accumulated charge carriers are expected to be in the $\alpha$-NPD side owing to the energy barrier at the interface. A possible origin of the difference in conductance is a local electric field formed by the permanent dipole moment in the vicinity of the interface, leading to a microscopic potential fluctuation [Fig. 12(b)]. Similar behavior has been reported as the gate dielectric dependence of charge mobility in OFETs, in which the dipolar disorder in the gate dielectric layer results in lower mobility. ${ }^{13-15}$ Note that as there is a small difference in the dielectric constant and its frequency dependence among the films, the observed phenomena should not be primarily attributed to the Fröhlich polaron. ${ }^{38}$

\section{v. CONCLUSION}

We demonstrate that the permanent dipole moment of a molecule and its orientational order play a significant role in the charge behavior at an organic heterointerface. DCM of bilayer devices consisting of $\alpha$-NPD films and several kinds of the other layer of polar or nonpolar molecular layers was conducted and the interface charge density, which appeared only in the device with polar molecules, was estimated. In addition, from the KP measurements of the corresponding bilayer structures, we found that GSP appeared in the films with polar molecules deposited on the $\alpha$-NPD film, indicating the orientational order of the permanent dipole moment in the resultant films. Higher interface charge density was found in the device containing the film with higher GSP. Although a quantitative disagreement still remains, our results strongly suggest that orientation polarization leads to interface charge in the actual devices. In addition, the $C-V$ and $C-f$ curves for the bilayer devices showed significant capacitance inflation if nonpolar molecules were used as the second layer. These results can be explained in terms of the dipolar disorder model: the presence of a permanent dipole moment results in microscopic potential fluctuation in the vicinity of the interface and suppresses the conductance of the accumulated holes along the interface in the adjacent layer.

As many organic semiconductor molecules possess permanent dipole moments, GSP and interface charge can more or less exist even in common organic semiconductor devices such as OLEDs. Interface charge is an important parameter, 
because it determines the least amount of accumulated charge in the operating device, which strongly relates to device efficiency and degradation. Moreover, the presence of a permanent dipole moment can suppress the charge spreading along organic heterointerfaces; thus, making an interface with polar molecules may lead to an efficient charge recombination and avoid cross-talk between neighboring pixels. Our results clearly demonstrate that the permanent dipole moment of the molecule and its orientational order in the evaporated film are important factors for understanding the charge accumulation properties of a device.

\section{ACKNOWLEDGMENTS}

We would like to thank Dr. H. Fukagawa (NHK) for useful suggestions for the UGH2 experiments. Y. Noguchi thanks members of Brütting's group (Universität Augsburg) for their kind cooperation in our experiments. We thank Nippon Steel Chemical Co., Ltd. for providing $\alpha$-NPD and $\mathrm{Alq}_{3}$ molecules. W.B. would like to thank the Japan Society for the Promotion of Science (JSPS) for an Invitation Fellowship for Research in Japan. This research is supported by JSPS through the "Funding Program for World-Leading Innovative R\&D on Science and Technology (FIRST Program)" initiated by the Council for Science and Technology Policy (CSTP), the Global-COE Project of Chiba University (Advanced School for Organic Electronics), and KAKENHI (Grant Nos. 21245042 and 22750167).

${ }^{1}$ G. G. Malliaras and J. C. Scott, J. Appl. Phys. 83, 5399 (1998).

${ }^{2}$ B. Ruhstaller, S. A. Carter, S. Barth, H. Riel, W. Riess, and J. C. Scott, J. Appl. Phys. 89, 4575 (2001).

${ }^{3}$ M. Matsumura, A. Ito, and Y. Miyamae, Appl. Phys. Lett. 75, 1042 (1999).

${ }^{4}$ F. Rohlfing, T. Yamada, and T. Tsutsui, J. Appl. Phys. 86, 4978 (1999).

${ }^{5}$ S. Berleb, W. Brütting, and G. Paasch, Synth. Met. 122, 37 (2001).

${ }^{6}$ T. Haskins, A. Chowdhury, R. H. Young, J. R. Lenhard, A. P. Marchetti, and L. J. Rothberg, Chem. Mater. 16, 4675 (2004).

${ }^{7}$ R. H. Young, C. W. Tang, and A. P. Marchetti, Appl. Phys. Lett. 80, 874 (2002).

${ }^{8}$ H. Aziz, Z. D. Popovic, N.-X. Hu, A.-M. Hor, and G. Xu, Science 283, 1900 (1999).

${ }^{9}$ D. Y. Kondakov and R. H. Young, J. Appl. Phys. 108, 074513 (2010).

${ }^{10}$ Y. Noguchi, Y. Tanaka, Y. Miyazaki, N. Sato, Y. Nakayama, and H. Ishii, in Physics of Organic Semiconductors, edited by W. Brütting and C. Adachi (Wiley-VCH) (in press).

${ }^{11}$ M. A. Baldo, Z. G. Soos, and S. R. Forrest, Chem. Phys. Lett. 347, 297 (2001).
${ }^{12}$ M. A. Baldo and S. R. Forrest, Phys. Rev. B 64, 085201 (2001).

${ }^{13}$ J. Veres, S. D. Ogier, S. W. Leeming, D. C. Cupertino, and S. M. Khaffaf, Adv. Funct. Mater. 13, 199 (2003).

${ }^{14}$ J. Veres, S. Ogier, G. Lloyd, and D. De Leeuw, Chem. Mater. 16, 4543 (2004).

${ }^{15}$ T. Richards, B. Matthew, and H. Sirringhaus, J. Chem. Phys. 128, 234905 (2008).

${ }^{16}$ Y. Noguchi, N. Sato, Y. Tanaka, Y. Nakayama, and H. Ishii, Appl. Phys. Lett. 92, 203306 (2008)

${ }^{17}$ W. Brütting, S. Berleb, and A. G. Mückl, Org. Electron. 2, 1 (2001).

${ }^{18}$ D. Y. Kondakov, J. R. Sandifer, C. W. Tang, and R. H. Young, J. Appl. Phys. 93, 1108 (2003).

${ }^{19}$ E. Ito, N. Hayashi, H. Ishii, N. Matsuie, K. Tsuboi, Y. Ouchi, Y. Harima, K. Yamashita, and K. Seki, J. Appl. Phys. 92, 7306 (2002).

${ }^{20}$ T. Manaka, K. Yoshizaki, and M. Iwamoto, Curr. Appl. Phys. 6, 877 (2006).

${ }^{21}$ N. Hayashi, K. Imai, T. Suzuki, K. Kanai, Y. Ouchi, and K. Seki, in IPAP Conf. Ser. 6, 69 (2004).

${ }^{22}$ M. Kröger, S. Hamwi, J. Meyer, T. Dobbertin, T. Riedl, W. Kowalsky, and H. Johannes, Phys. Rev. B 75, 235321 (2007).

${ }^{23}$ N. Kajimoto, Ph.D. dissertation, Tokyo Institute of Technology, 2008.

${ }^{24}$ D. Y. Kondakov, J. Appl. Phys. 99, 024901 (2006).

${ }^{25}$ A. P. Marchetti, K. E. Sassin, R. H. Young, L. J. Rothberg, and D. Y. Kondakov, J. Appl. Phys. 109, 0137091 (2011).

${ }^{26}$ Y. Tanaka, Y. Noguchi, M. Kraus, W. Brütting, and H. Ishii, Org. Electron. 12, 1560 (2011).

${ }^{27}$ S. Egusa, A. Miura, N. Gemma, and M. Azuma, Jpn. J. Appl. Phys., Part 1 33, 2741 (1994).

${ }^{28} \mathrm{~S}$. Ogawa, Y. Kimura, H. Ishii, and M. Niwano, Jpn. J. Appl. Phys., Part 2 42, L1275 (2003).

${ }^{29}$ S. Nowy, W. Ren, A. Elschner, W. Lövenich, and W. Brütting, J. Appl. Phys. 107, 054501 (2010).

${ }^{30}$ Y. Noguchi, N. Sato, Y. Miyazaki, and H. Ishii, Appl. Phys. Lett. 96, 143305 (2010).

${ }^{31}$ S. T. Lee, Y. M. Wang, X. Y. Hou, and C. W. Tang, Appl. Phys. Lett. 74, 670 (1999).

${ }^{32}$ N. Sato, Y. Noguchi, Y. Tanaka, Y. Nakayama, and H. Ishii, Proc. SPIE 7051, 1S (2008).

${ }^{33}$ K. Yoshizaki, T. Manaka, and M. Iwamoto, J. Appl. Phys. 97, 023703 (2005).

${ }^{34}$ K. Sugi, H. Ishii, Y. Kimura, M. Niwano, E. Ito, Y. Washizu, N. Hayashi, Y. Ouchi, and K. Seki, Thin Solid Films 464-465, 412 (2004).

${ }^{35}$ N. Kajimoto, T. Manaka, and M. Iwamoto, Jpn. J. Appl. Phys., Part 1 46, 2740 (2007).

${ }^{36}$ K. Ozasa, S. Nemoto, T. Isoshima, E. Ito, M. Maeda, and M. Hara, Appl. Phys. Lett. 93, 263304 (2008).

${ }^{37}$ M. Schwoerer and H. Wolf, Organic Molecular Solids (Wiley-VCH, 2007).

${ }^{38}$ I. N. Hulea, S. Fratini, H. Xie, C. L. Mulder, N. N. Iossad, G. Rastelli, S. Ciuchi, and A. F. Morpurgo, Nature Mater. 5, 982 (2006).

${ }^{39}$ Y. Nakayama, S. Machida, Y. Miyazaki, T. Nishi, Y. Noguchi, and H. Ishii, "Electronic structures at organic heterojunctions of N,N'-bis(1naphthyl)-N, $\mathrm{N}^{\prime}$-diphenyl-1,1'-biphenyl-4,4'-diamin (NPB)-based organic light emitting diodes," (unpublished).

${ }^{40}$ I. G. Hill and A. Kahn, J. Appl. Phys. 86, 2116 (1999). 